LBL- -29183

DE91 004414

\title{
Evaluation of the X-Ray Response of a Position-Sensitive Microstrip Detector with an Integrated Readout Chip
}

C. Rossington, J. Jaklevic, C. Haber and H. Spieler

Lawrence Berkeley Laboratory

University of California

Berkeley, CA 94720

and

J. Reid

Department of Physics

University of California

Davis, CA 95616

(current address: Department of Applied Physics,

California Institute of Technology, Pasadena, CA 91125)

This work was supported by the Director's Office of Energy Research, Office of Health and Environmental Research, U.S. Department of Energy under Contract No. DE-ACO3$76 \mathrm{SF} 00098$. 
LBL-29183

\author{
EVALUATION OF THE X-RAY RESPONSE OF A POSITION-SENSITIVE \\ MICROSTRIP DETECTOR WITH AN INTEGRATED READOUT CHIP
}

\author{
C. Rossington, J. Jaklevic, J. Reid*, C. Haber, \\ ana $H$. Spieler \\ Lawrence Berkeley Laboratory, \\ University of California, Berkeley, CA 94720 \\ *Department of Physics, \\ University of California, Davis, CA 95616 \\ (Current address: Department of Applied Physics, \\ California Institute of Technology, \\ Pasadena, CA 91125)
}

\title{
INTRODUCTION
}

Many of the scientific programs proposed for implementation at existing and future synchrotron radiation sources involve measurement of the spatial distribution of transmitted or diffracted $x$-ray beams. The design of a general purpose positionsensitive detector for use in such experiments must address several conflicting requirements. Ideally, such a detector would meet the following criteria: high detection efficiency over a wide energy range, large dynamic range in measured photon intensities and response to a wide range of measurement intervals. Existing detector systems only partially meet these requirements, although much work has gone into improving the current technology.

Considerable research has been directed toward adapting twodimensional semiconductor charge coupled diode (CCD) arrays, originally developed for visual and infrared spectroscopy, for the detection of $x$-rays.1-4 The small pixel size (as small as 10 $x 10 \mu \mathrm{m}$ ) and large array dimensions (on the order of $500 \times 500$ pixels) of a CCD result in high spatial resolution. In addition, modern CCD's are capable of excellent energy resolution $(80 \mathrm{eV} \text { at } 1.5 \mathrm{keV} \text { and } 150 \mathrm{eV} \text { at } 5.9 \mathrm{keV})^{3}$ and a large dynamic range on the order of $10^{4} .4$ Their drawbacks include: (1) pixel sizes that are too small for optimum diffraction works, (2) low quantum efficiency at $x$-ray energies greater than $5 \mathrm{keV}$ due to the 10 - $15 \mu \mathrm{m}$ thickness of the device, and (3) position and pulse amplitude ambiguities for the portion of $x$-ray events that occur in the undepleted region of the device. The CCD readout scheme requires pixel-to-pixel transfer of charge to one output channel, which limits the data acquisition rate to approximately 
I $s$ for a $584 \times 390$ array. ${ }^{4}$ This precludes the use of CCD's in experiments requiring fast data collection for short time resolution. CCD's suffer significant radiation damage at doses $>1 C^{5}$ rad, which limits their use in the harsh environment of a synchrotron.6,7

Silicon photodiode arrays (PDA), developed for the detection of visible light, have been successfully utilized in the detection of $x$-rays in EXAFS (extended $x$-ray absorption fine structure) experiments. 8 These one-dimensional arrays typically contain 1024 strips, each $2.5 \mathrm{~mm}$ long and $15-100 \mu \mathrm{m}$ wide, fabricated on $300 \mu \mathrm{m}$ thick high resistivity silicon. PDA's suffer less radiation damage than $C C D$ 's, and hence have typically been chosen over CCD's for synchrotron environments. 6 Like the CCD, PDA's must be cooled to keep the dark current at low levels and there is only crne output channel for readout of all the strips. The readout time per detector element of a one-dimensional PDA is comparable to a CCD, but the reduced number of elements results in faster total readout times of the order of milliseconds. The advantages of a PDA include: (1) they are relatively thick, resulting in efficient detection of higher energy x-rays, (2) they operate in a fully depleted mode, thus eliminating the signal tailing from partial charge collection which occurs in the undepleted region of a CCD, and (3) they exhibit a high dynamic range on the order of $10^{4}$. A disadvantage is that they operate in a charge integrating mode in which there is no energy discrimination. In principal, they can be adapted for use with low-noise pulse processing electronics for energy discrimination, but the expense of multiple preamplifiers and shaping amplifiers precludes this option.

Numerous hybrid approaches involving phosphors to convert the $x$-ray radiation to visible light, which is then efficiently detected by a CCD or PDA, have also been demonstrated.5,9,10 These hybrids mitigate some of the drawbacks associated with direct detection, but increase the complexity and expense of the system, and generally result in loss of energy discrimination.

In this paper, a position-sensitive silicon microstrip detector and its compatible integrated readout chip are described as an alternative to the above mentioned detectors. The detector and readout chip, together called the silicon Vertex Detector (SVX), were adapted from an existing system designed at LBL for charged particle detection at the Collider Detector Facility at Fermilab.11 The interest in this particular strip detector system for $x$-ray applications arises from its dual energy and spatial discrimination capabilities. In addition, it has the unique capability of so-called "sparse readout", in which only those detector strips with pertinent event information are read out. The sparse readout mode enables fast, efficient management of data from low occupancy events. The sparse readout capability is especially attractive for x-ray diffraction applications, in which only a minority of the detector strips contain useful information. We present a description of the svx microstrip detector and the results of some preliminary investigations using a laboratory $x$-ray source. 


\section{DETECTOR AND CHIP DESCRIPTION}

Figure 1 is a schematic of the silicon microstrip detector wire-bonded to its compatible readout chip. The detector was fabricated on $280 \mu \mathrm{m}$ thick high resistivity silicon using a process developed at LBI which significantly minimizes detector leakage currents.12 There are 128 detector strips, each defined by the entrance window $\mathrm{p}^{+}$boron-diffused contacts. A single $\mathrm{n}^{+}$ contact. on the backside consists of phosphorus-diffused polysilicon. This particular detector has $15 \mu \mathrm{m} \times 6.3 \mathrm{~mm}$ strips on a $55 \mu \mathrm{m}$ pitch, as shown in the cross-section of Fig. 2, but the specific dimensions can be varied somewhat as required, so long as the detector strip pitch is compatible with the pitch of the output channels on the readout chip. The detector is reversebiased to full depletion, resulting in virtually complete charge collection of all $x$-ray events occurring in the $280 \mu \mathrm{m}$ thick active volume. Each detector strip is individually wire-bonded to its corresponding pulse processing circuitry on the integrated readout chip. (Recently, a process was developed to fully integrate the detector and readout chip on the same wafer, eliminating the need for wire-bonding ${ }^{13}$ ). The readout chip consists of 128 identical data acquisition channels, each comprised of a preamplifier, pulse shaper and threshold comparator. Multiplexing circuitry on the chip selects those channels that register hits and directs their analog signal and digital address to a single output line.11

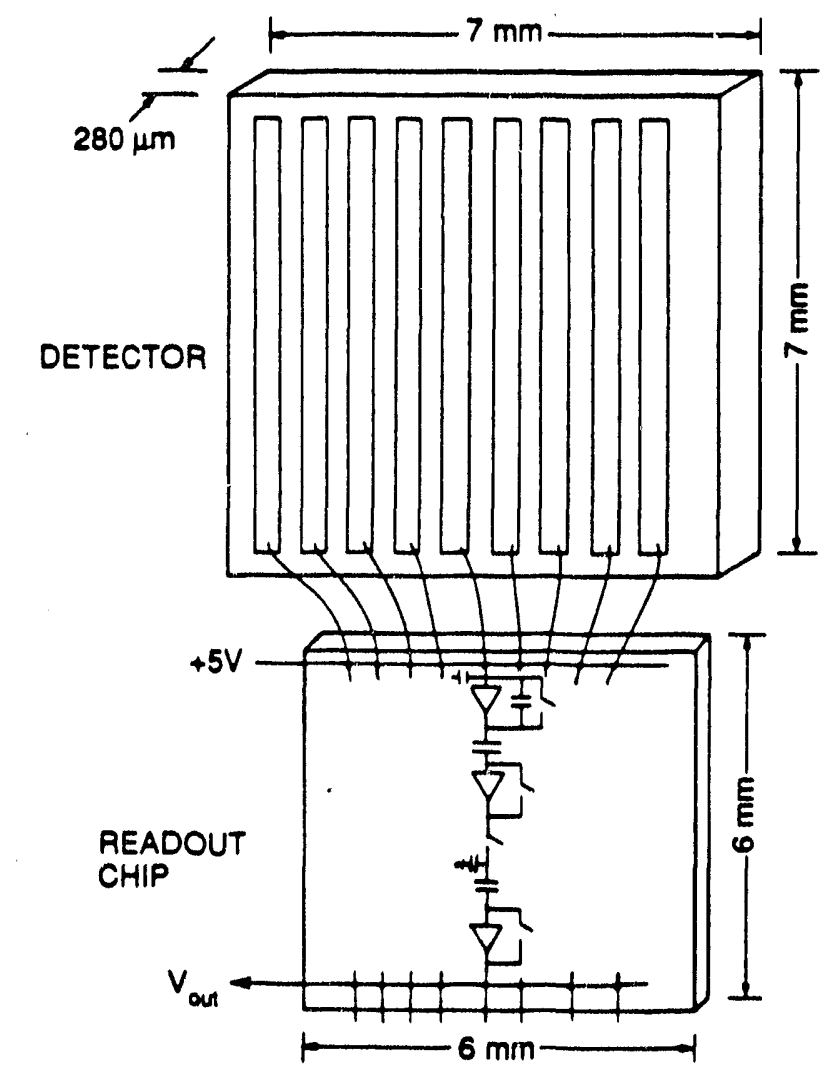

XBL $907-6465 B$

Eig. I Schematic of an SVX microstrip detector wire-bonded to an integrated readout chip. Only a few of the $128 \mathrm{de}-$ tector strips are shown, and only a portion of one of the 128 pulse-processing channels on the readout chip is shown. 


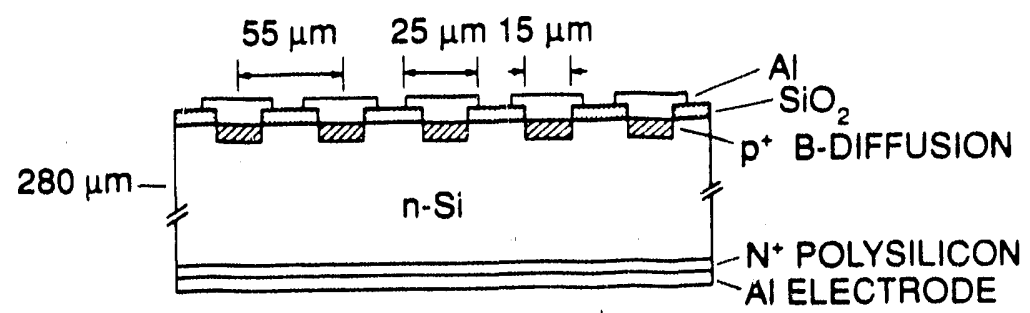

xacorat

Fig. 2 Schematic of the cross-section of the SVX detector.

The detector and readout chip were enclosed in a light-tight box with a $50 \mu \mathrm{m}$ thick Be window. A block diagram summarizing the relationship between the detector, one pulse processing channel on the readout cirip and the data acquisition system is shown in Fig. 3. The conicrol of the readout chip, data acquisition and data manipulation, are accomplished using an Apple Macintosh SE personal computer interfaced with a General Purpose Interface Bus (GPIB) to a CAMAC-based data acquisition system.14 A CAMAC pattern generator module is used to produce programmable patterns of signals for the timing and control of the switches in the analog and digital circuitry of the readout chip. A second CAMAC module receives, digitizes and stores the analog and digital data from the chip. The type of readout mode desired dictates what kind of chip control program is selected and downloaded to the CAMAC pattern generator from the computer. The custom PC program, written specifically for the Macintosh, operates in a multi-channel analyzer.mode allowing real-time observation of the data in one of the following three forms: pulse height distribution as a function of energy for a specific detector strip, (2) total number of events per strip as a function of strip number, and (3) total energy per strip as a function of strip number.

As stated previously, the design of the readout chip allows for several types of data acquisition. The two types which are described here, and which are most relevant to $x$-ray work, are sparse readout and full readout. In the sparse mode, only those strips containing event information above (or below) a preset threshold level are readout. For readout of an event above a minimurn threshold level, the stored signal charge must be larger than the stored threshold charge to latch the analog comparator. When the analog comparator latches, it activates the digital section for transfer of strip and chip identification numbers to the digital bus and allows transfer of the analog information to the analog bus. However, if the signal charge is less than the threshold charge, the comparator does not latch and no readout occurs. It is important to note that the analog signal in the sparse mode is equal to the difference between the input signal and the threshold signal. To achleve full energy discrimination the value of the threshold voltage must be added back via the software. Full energy discrimination is readily achieved in the full readout mode, in which there is no preset threshold and the entire input signal is transferred to the analog bus. In the full readout mode, all 128 strips are read out after each cycle, requiring approximately $500 \mu \mathrm{s}$. The readout rate of the sparse mode is approximately $20 \mu \mathrm{s}$ plus $4 \mu \mathrm{s}$ par strip, thus the total 
time depends on the number of strips with event information. The readout rates are significantly faster than CCD's or PDA's, even in the full readout mode.

Fully exploiting the capabilities of the readout chip requires that the signal source be on only during a specific integration time interval of the chip control program. A pulsed $x-$ ray tube, triggered by the chip control program just prior to the signal integration time, was used for this work. An x-ray pulse width of $1-2 \mu s$, in conjunction with integration times of 2 - $6 \mu \mathrm{s}$, were used. In the full readout mode there is one time window during which the signal charge is integrated. In the sparse readout mode there are two integration time windows, one for integration of the signal charge and one for the threshold charge. For accurate comparison between the threshold and signal charges, it is critically important that each occur only during its respective time window.

One of the advantages of the present detector design is that it is not limited in thickness. As the curves in Fig. 4 show, the $280 \mu \mathrm{m}$ thick SVX detector is much more efficient at energies $>3 \mathrm{keV}$ than a $15 \mu \mathrm{m}$ thick CCD. For example, at $10 \mathrm{keV}$ the SVX is ten times more efficient than the CCD. In the present study an Rh-anode $x$-ray tube, with average outpli: $20.5 \mathrm{keV}$, was used to characterize the $x-r a y$ response of the svx detector. The response of the detector in both the sparse and full readout modes and in a simple diffraction experiment will be described.

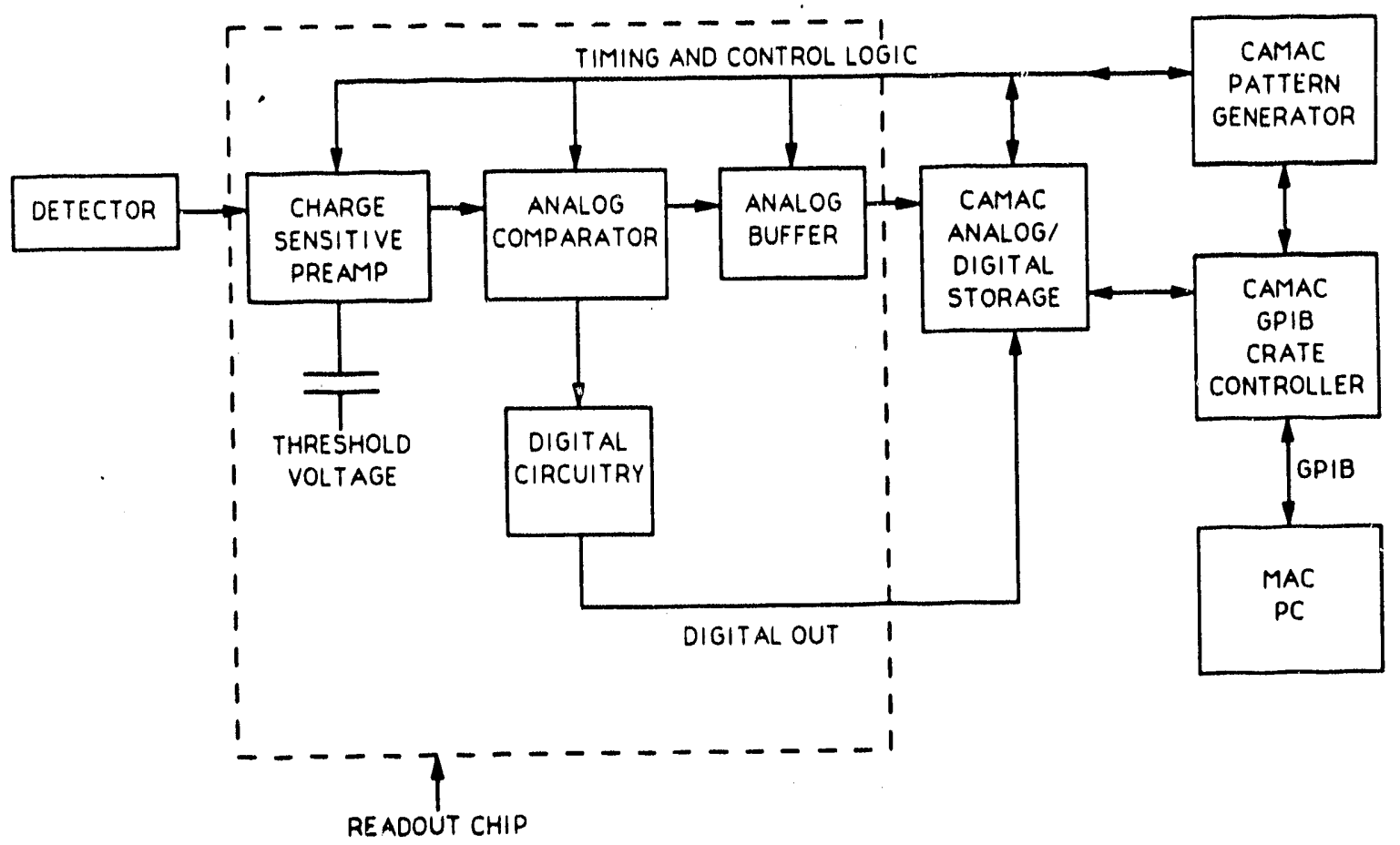

XBL $908-2902$

Fig. 3 Block diagram showing the relationship between the detector, one channel of the readout chip, the CAMAC based data acquisition system and the computer. 


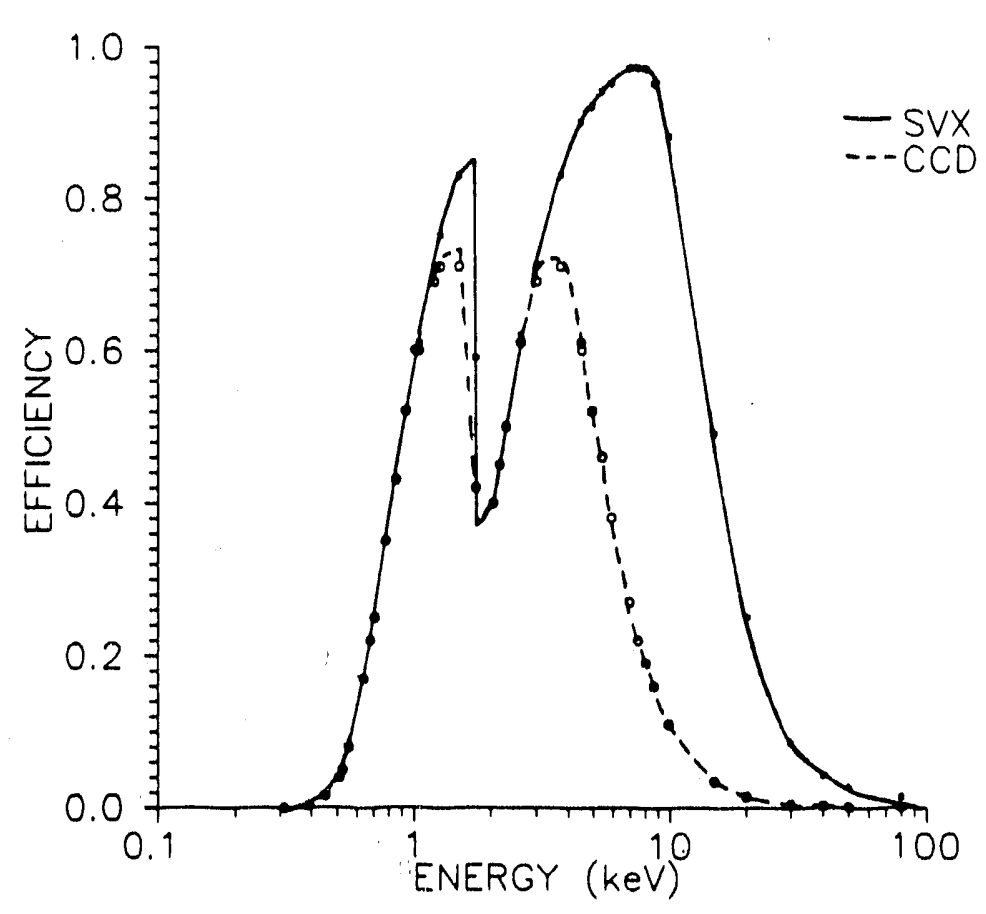

$x \geq 2900-2900$

Eig. 4 Efficiency as a function of energy for a typical $280 \mu \mathrm{m}$ thick SVX detector compared with a front-iliuminated 15 $\mu m$ thick CCD. The low energy data was calculated assuming a thin entrance window consisting of an Al electrode and implanted Si.

\section{RESULTS}

The response of the SVX detector to $R h x$-rays and to a calibrated injected pulse in the full readout mode is shown in Fig. 5. The $R h K x$-rays are centered at $\sim 20 \mathrm{keV}$ and are observed as a high energy shoulder on the background noise peak. The electronic noise is on the order of $4.5 \mathrm{keV}$ FWHM at $\sim 41 \mathrm{keV}$, as calculated from the calibrated injected pulse. The FWHM of the Rh $x$-ray distribution is on the order of $12 \mathrm{keV}$. This distribution includes both the $R h K \alpha$ and $K \beta$ emission lines and some contribution from the Bremsstrahlung continuum. Other SVX detector systems have exhibited better energy resolution, of the order of 2 $\mathrm{keV}$ FWHM at $60 \mathrm{keV}^{12}$, as will be discussed later.

As can be seen from Fig. 5, the number of counts in the noise peak is significantly greater than the number of counts in the $x$-ray peak when operating in the full readout mode. This is a consequence of reading out all the strips during each cycle, which results in the noise being read out when there are no $x-$ ray events. In this readout mode one cannot simply do real time observation of the total number of ccunts per detector strip as a quick and efficient way of determining which strips are being hit by $x$-rays. In contrast, the sparse readout mode is ideally suited for fast identification of the strips with x-ray events, since the comparator suppresses noise events. The data of Fig. 6 were taken in the sparse readout mode and show the total number of counts per strip as a function of strip number and as a function of the preset threshold level. A $40 \mu \mathrm{m}$ wide slit was placed $\sim 1 / 2$ inch from the detector, parallel to strip number 19, 
to achieve single strip illumination. From Fig. 6, it is clear that strip number 19 appears no different than other strips not being hit by $x$-rays, until a threshold voltage of $8 \mathrm{mV}$ is reached. A minimum of $8 \mathrm{mV}$ (equivalent to $\sim 11 \mathrm{keV}$ for this device) is required to subtract the noise from the $x$-rays events. The larger the signal-to-noise ratio for the device, the lower is the threshold voltage required to subtract the noise from the signal. Increasing the threshold level beyond 8 $\mathrm{mV}$ reduces the number of strips read out with noise events, but also significantly reduces the $x$-ray signal intensity. As can be seen in Fig. 6 , the strips adjacent to strip 19 have approximately one-tenth the number of $x$-ray events as does strip 19. These are due to lateral diffusion of charge carriers from $x$-ray events occurring in the shallow field-free region between the 15 $\mu \mathrm{m}$ wide $\mathrm{p}^{+}$strips. With accurate collimation of the $\mathrm{x}$-ray beam to only one strip width, the spatial resolution should be equivalent to the strip pitah for normal incidence $x$-rays.

The detector was operated in both the full and sparse readout modes in a simple diffraction experiment. A (200) Lif crystal was used to diffract the $R h K \alpha_{1}$ and $K \alpha_{2}$ lines, with Bragg angles of $8.75^{\circ}$ and $8.826^{\circ}$, respectively. These two $K \alpha$ lines are separated by approximately $400 \mu \mathrm{m}$ at a distance of $15 \mathrm{~cm}$ from the LiF crystal, corresponding to an SVX detector strip separation of 7-8 strips. Figure 7 shows the total number of counts per strip at a distance of $15 \mathrm{~cm}$ from the diffracting crystal where the individual $K \alpha_{1}$ and $k \alpha_{2}$ lines are clearly distinguished. This particular data was taken in the sparse readout mode, reading out only those strips with events occurring above the preset threshold level. Alternatively, the full readout mode can be used, yielding spectra identical to those of $\mathrm{Fig} .7$ and in addition yielding pulse height distributions for each strip, but it is much less efficient.

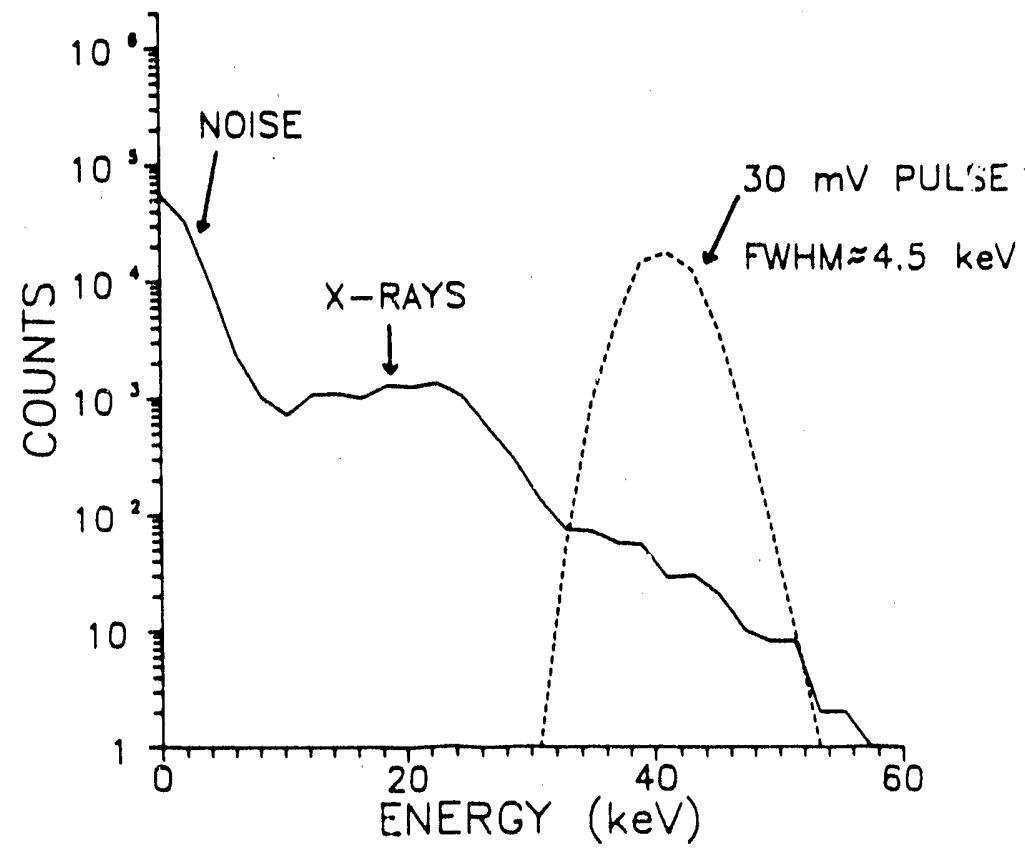

$X=2000-2800$

Fig. 5 Response of a typical svx detector strip to $R$ h $K$ x-rays and to a calibrated injected pulse, using the full readout mode. 


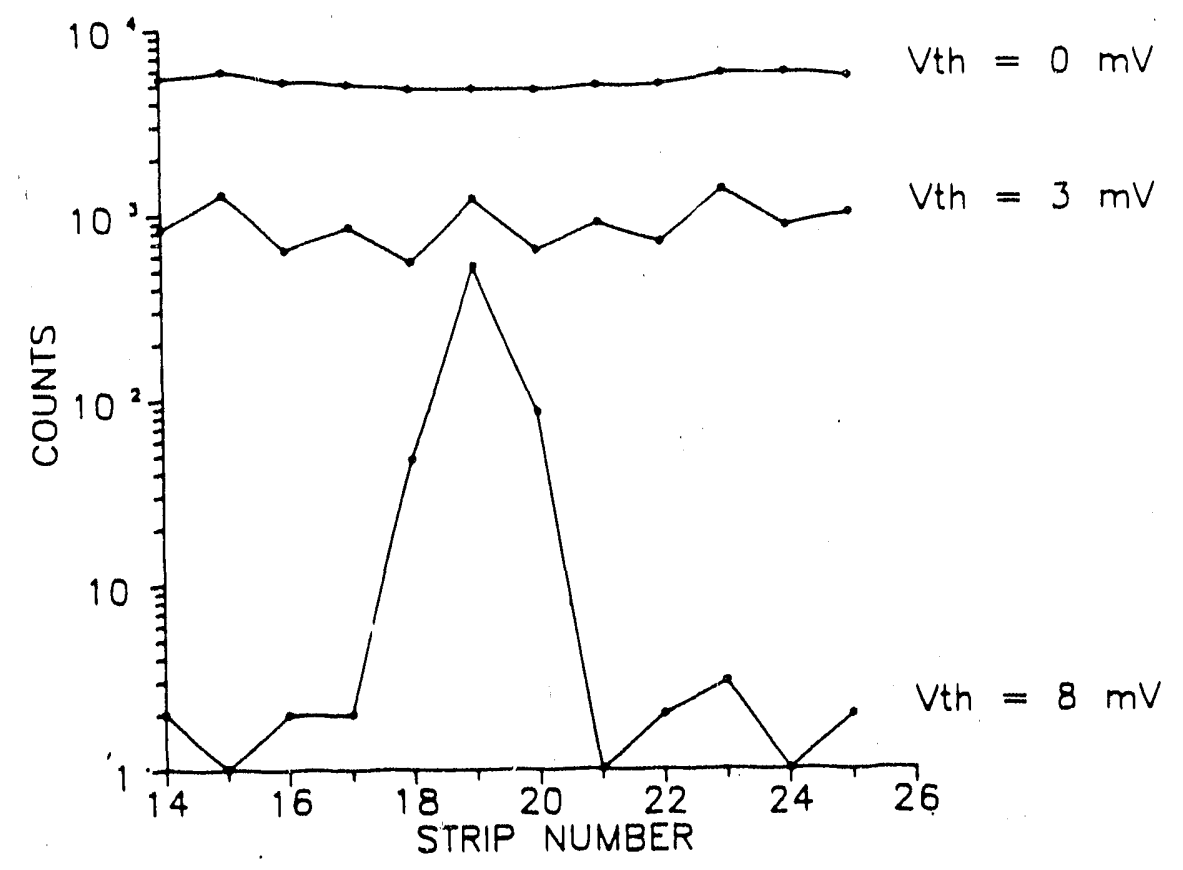

$x 02,908-2901$

Fig. 6 Total counts per strip in the sparse mode as a function of threshold voltage, vth, when $x$-rays are hitting only strip rumbers 18,19 and 20 .

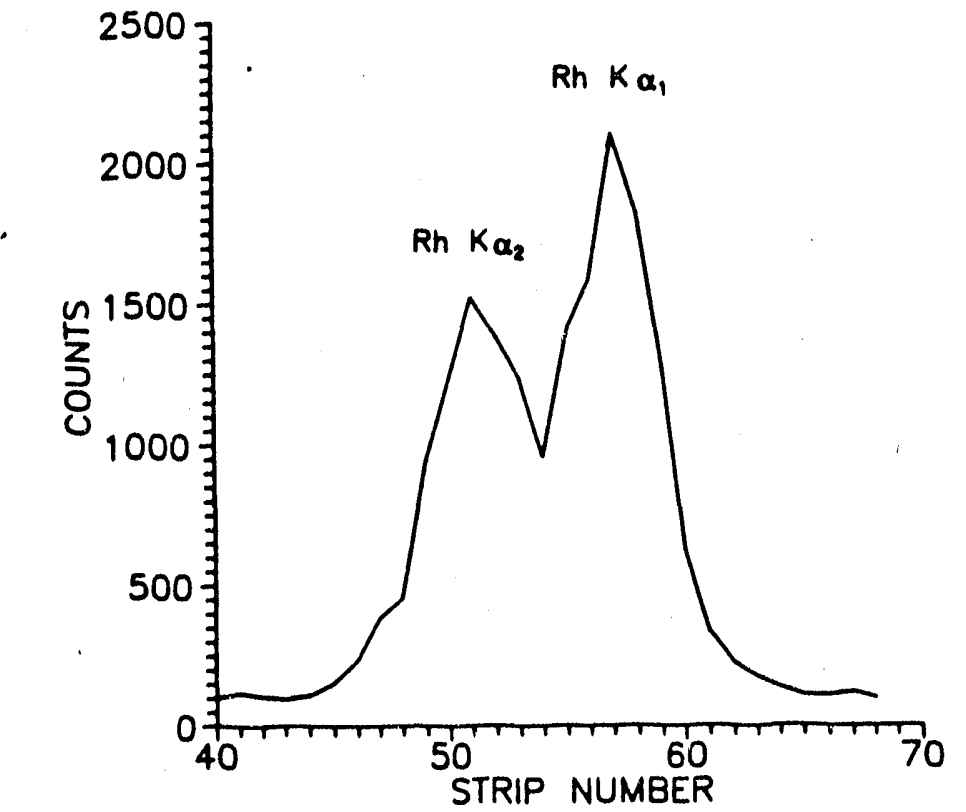

xan $900-2008$

Fig. 7 Diffraction of $R h k \alpha_{1}$ and $k \alpha_{2} x$-rays from a (200) LiF crystal. Total counts as a function of strip number for a distance of $15 \mathrm{~cm}$ between the detector and IiF.

\section{DISCUSSION}

The SVX microstrip detector offers several advantages over the currently available position-sensitive detector technology, although further development is required to bring it to its theoretical potential for $x$-ray detection. Data taken on other SVX 
systems in high energy physics experiments, in which the minimum ionizing particle is $\sim 90 \mathrm{keV}$, indicate that better performance should be possible in $x$-ray applications and further optimization is necessary. The SVX readout chip (version C''') used in this work has exhibited an electronic noise as low as 2200 electrons ( $1.7 \mathrm{keV}$ FWHM) in previous measurements. Additional detectors and readout chips will be tested in an effort to determine the source of variability in the noise. In addition, measurements on the recently developed monolithically integrated detector and preamplifier indicate that an electronic noise of 80 electrons at $8 \mu \mathrm{s}$ integration time is obtainable. It is planned to evaluate one or more of these systems and also a $16 \mathrm{x}$ 16 pixel array, in response to $R h$ as well as lower energy $x-$ rays.

Preliminary measurements indicate there is little or no lateral diffusion or cross-talk among detector strips and the spatial resolution should be equivalent to the strip pitch for normal incidence $x$-rays. The fully depleted detector allows for virtually complete charge collection from all x-ray events occurring in the active volume, in contrast to a CCD which exhibits partial charge collection from events occurring in the undepleted portion of the device.

The readout chip is capable of full readout of all 128 strips every $500 \mu \mathrm{s}$, thus a maximum of 2000 x-ray events per strip per second can theoretically be handled by the chip in this mode. Higher count rates can be achieved using the sparse readout mode. For example, if only three strips were being hit by $x-$ rays in a diffraction experiment, the readout rate would be approximately $32 \mu \mathrm{s}$, allowing reallout of $30,000 \mathrm{x}$-ray events per strip per second. The count rates observed here were $2-3$ orders of magnitude less than that, due to inefficient data management by the computer and software. Several things can be done to increase the data acquisition rate, such as: (1) utilize a faster computer, (2) exchange the slow GPIB bus for a faster bus, and (3) read out the digital storage buffer in the CAMAC data acquisition module only when full as opposed to after each readout cycle. To achieve reasonable data accumulation rates in practical $x$-ray experiments and especially for time-resolved experiments, all three of the above changes should be made.

As stated previously, the signal source must be synchronized with the readout chip control program for this chip so that the signal is integrated during the appropriate time interval. It is possible, when operating in the full readout mode, that this requirement be relaxed, but some degradation of the energy resolution will occur. However, this requirement is mandatory in the sparse mode. If the svx detector were to be used in conjunction with a synchrotron $x$-ray source, the readout control program could be triggered appropriately in time with the period of the synchrotron. The readout chip control program could also be modified to allow triggering of the program every 100 or so cycles of the synchrotron and to suppress charge collection from $x$-ray events occurring during cycles between triggering. 
The effects of a synchrotron radiation environment on the SVX detector and chip are as yet unknown. Radiation damage studies have been performed on the SVX chip using a Co60 source, neutrons and protons, with a corresponding loss of gain and some increase in noise at radiation levels of 30 - $50 \mathrm{Krad} .15$ A radiation-hard version of the SVX chip has been designed and fabricated for high energy physics applications that will provide indications of potential radiation resistance in $x$-ray fields.

In principal, the readout chip could be used with other detector configurations, such as arrays, and other detector materials. Thicker silicon detectors, or detectors fabricated from germanium, would afford devices of much greater efficiency than the one described here. Cooling the SVX detector could also yield some advantages in lower detector and electronic noise for better energy resolution.

\section{SUMMARY}

The performance of an SVX siiicon microstrip detector and its compatible integrated readout chip have been evaluatied in response to $R h K \alpha$-rays (average energy $20.5 \mathrm{keV}$ ). The energy and spatial discrimination capabilities, efficient data management and fast readout rates make it an attractive alternative to the CCD and PDA detectors now being offered for x-ray position sensitive diffraction and EXAFS work. The sVX system was designed for high energy physics applications and thus further development of the existing system is required to optimize it for use in practical $x$-ray experiments. For optimum energy resolution the system noise must be decreased to its previously demonstrated low levels of $2 \mathrm{keV}$ FWHM at $60 \mathrm{keV}$ or less, and the data handling rate, of the computer must be increased. New readout chips are now available that offer the potential of better performance.

\section{REFERENCES}

1. R. Stern, K. Liewer and J. Janesick, Evaluation of a virtual phase charge-coupled device as an imaging $x$-ray spectrometer, Rev. Sei. Instrum., 54(2):198 (1983).

2. J. Janesick, T. Elliott, J. MCCarthy, H. Marsh, S. Collins and $M$. Blouke, Present and future CCD's for UV and X-ray scientific measurements, IEEE Trans. Nucl. Sci, NS$32(1): 409$ (1985).

3. J. Janesick, T. Elliott, R. Bredthauer, C. Chandler and B. Burke, Fano-noise-limited CCD's, "Optoelectronic Applied Science and Engineering Symposium", X-ray Instrumentation in Astronomy, San Diego, Aug. 14-19, 1988.

4. B. Rodricks, R. Clarke, R. Smither and A. Fontaine, A virtual phase CCD detector for synchrotron radiation applications, Rev. Sci. Instrum., $60(8): 2586$ (198.9). 
5. U. Arndt, X-ray position-sensitive detectors, J. AppI. Cryst, 19:115 (1986).

6. B. Magorrian and N. Allinson, Soft $x$-ray damage in CCD detectors, Nucl. Instrum. Methe, A273:599 (1988).

7. J. Janesick, T. Elliot and F. Pool, Radiation damage in scientific charge-coupled devices, IEEE Trans. Nucl. Sci, $36(1): 572$ (1989).

8. N. Allinson, G. Baker, G. Greaves and J. Nicoll, PDA system for energy dispersive EXAFS, Nucl. Instrum. Methe, A2 66:592 (1988).

9. N. Allinson and G. Greaves, Hybrid linear array detectors for synchrotron radiation applications, Nucl. Instrum. Meth., A273:620 (1988).

10. H. Fuchs, D. Wu and B. Chu, An area x-ray detector system based on a commercially available CCD unit, Rev. Sci. Instrum, $61(2): 712$ (1990).

11. S. Kleinfelder, W. Carithers, Jr., R. Ely, Jr., C. Haber, F. Kirsten and H. Spieler, A flexible 128 channel silicon strip detector instrumentation integrated circuit with sparse data readout, IEEE. Trans. Nucl. Sci, 35(1):171 (1988).

12. S. Holland, Fabrication of detectors and transistors on highresistivity silicon, Nucl. Instrum. Meth, A275:537 (1989).

13. S. Holland and H. Spieler, A monolithically integrated detector-preamplifier on high resistivity silicon, IEEE Trans. Nucl. Sci, $37(2): 463$ (1990).

14. F. Kirsten and $C$. Haber, $A$ versatile, programmable cnntrol and data acquisition system for complex integrated circuits, IEEE Trans. Nucl. Scin, $37(2): 288$ (1990).

15. R. Yarema, D. Amidei, T. Bohn, T. Zimmerman, R. Ely, C. Haber, S. Kleinfelder, W. Wester and S. Vejcik, Radiation effects on the SVX silicon strip readout chip, IEEE Trans. Nucl. Sci, $37(2): 434$ (1990).

\section{ACKNOWLEDGMENT / DISCLAIMER}

The authors would like to thank steve Holland at Lawrence Berkeley Laboratory for the faurication of the detector. This work was supported by the Direc'or's Office of Energy Research, Office of Health and Environmental Research, U.S. Department of Energy under Contract No. DE-AC03-76SF00098. Reference to a company or product name does not imply approval or recommendation of the product by the University of California or the U.S. Department of Energy to the exclusion of others that may be suitable. 

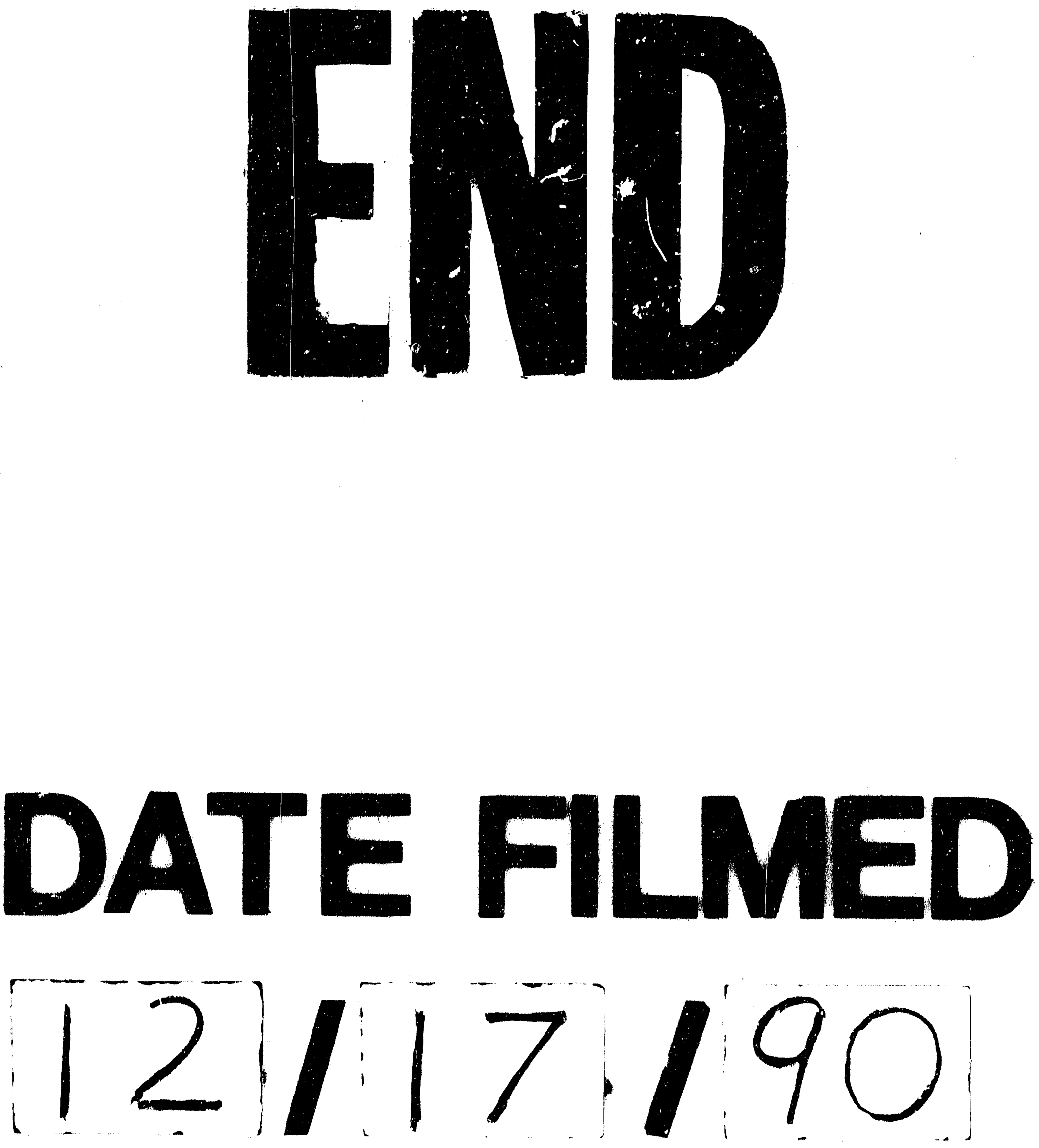
